\title{
BMJ Open Quantifying the hospitalised morbidity and mortality attributable to traumatic injury using a population-based matched cohort in Australia
}

\author{
Rebecca J Mitchell, ${ }^{1}$ Cate M Cameron, ${ }^{2}$ Rod McClure ${ }^{3}$
}

To cite: Mitchell RJ, Cameron CM, McClure R. Quantifying the hospitalised morbidity and mortality attributable to traumatic injury using a populationbased matched cohort in Australia. BMJ Open 2016;6: e013266. doi:10.1136/ bmjopen-2016-013266

- Prepublication history for this paper is available online. To view these files please visit the journal online (http://dx.doi.org/10.1136/ bmjopen-2016-013266).

Received 1 July 2016 Revised 6 November 2016 Accepted 8 November 2016

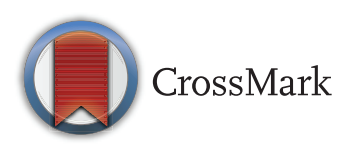

\section{${ }^{1}$ Australian Institute of Health Innovation, Macquarie University, Australia ${ }^{2}$ Menzies Health Institute Queensland, Griffith University, Nathan, Queensland, Australia ${ }^{3}$ Harvard Injury Control Research Center, Harvard School of Public Health, Harvard University, Cambridge, Massachusetts, USA}

Correspondence to Dr Rebecca Mitchell; r.mitchell@mq.edu.au

\section{ABSTRACT}

Objectives: To quantify the 12-month hospitalised morbidity and mortality attributable to traumatic injury using a population-based matched cohort in Australia.

Setting: New South Wales, Queensland and South Australia, Australia.

Participants: Individuals $\geq 18$ years who had an injury-related hospital admission in 2009 formed the injured cohort. The non-injured comparison cohort was randomly selected from the electoral roll and was matched 1:1 on age, gender and postcode of residence at the date of the index injury admission of their matched counterpart.

Primary outcome measures: Using linked emergency department presentation, hospital admission and mortality records from 1 January 2008 to 31 December 2010 for both the injured and noninjured cohorts, 12-month mortality and pre-index and post-index injury hospital service use was examined. Adjusted rate ratios and attributable risk were calculated.

Results: There were 167600 individuals injured in 2009 and admitted to hospital in New South Wales, South Australia or Queensland with a matched comparison. The injured cohort had 3 times higher proportion of having $\geq 1$ comorbidity preinjury, higher preinjury hospital service use, and a higher 12-month mortality compared with a non-injured comparison group. The injured cohort had $2.20(95 \% \mathrm{Cl} 2.12$ to 2.28) times higher rate of hospital admissions in the 12 months post the index injury admission compared with the non-injured comparison cohort. Injury was a likely contributory factor in at least $55 \%$ of hospitalisations within 12 months of the index injury hospitalisation.

Conclusions: Individuals who had an injury-related hospitalisation had higher mortality and are hospitalised at increased rates for many months postinjury. While comorbid conditions are significant, they do not account for the differences in outcomes. This study contributes to informing research efforts on better quantifying the attributable burden of hospitalised injury-related disability and mortality in Australia.

\section{Strengths and limitations of this study}

- Uses information from population-based data collections from three Australian states.

- Uses a novel 1:1 matched pair cohort design to compare injury outcomes within a 12-month period pre and post an index injury hospitalisation.

- However, the study is likely to underestimate comorbidities experienced by individuals, as comorbidities were only able to be identified in hospitalisation data.

\section{INTRODUCTION}

Accurately measuring the burden of injury is important for priority-setting and for monitoring the impact of injury prevention initiatives. ${ }^{2}$ The Australian and Global Burden of Disease and Injury studies use results from injury incidence and outcome research to quantify the injury burden, ${ }^{1}{ }^{3}$ with the quality of the Global Burden of Disease and Injury studies estimates being reliant on the strength of the evidence provided from these studies. Much of the existing injury outcome research is based on inception cohort studies. $^{2}{ }^{4-6}$ Use of a matched pair cohort design to control for health status preceding the injury event and isolate injury attributable postinjury outcomes are rare.

The few injury outcome studies that have included randomly selected, matched noninjured comparison groups have found that, postinjury, injured individuals experience higher health service use, in terms of hospital admissions, general practitioner or physician visits, use of physiotherapy and use of home care services than their non-injured counterparts. ${ }^{7-10}$ Further, previous matched pair cohort studies have shown both that an individual's recovery from injury can be influenced by the presence of pre-existing 
comorbid conditions, ${ }^{11-14}$ and that postinjury health status is a combination of injury-related outcomes and background health burden suffered by the patient that is higher among injured people but is unrelated to the incident injury being evaluated. By overlooking the effect of comorbidities, inception cohorts of a series of injury cases (which matched controls) overestimate the true burden of injury. Case series cohorts that do not use a matched cohort control frequently attempt to control for comorbidity using patient self-reports of their preinjury health status, or normative health status date from the general population. However, both these methods have been shown to introduce recall and selection biases. ${ }^{7}{ }^{15}$ These biases are avoided by use of a matched pair design and record linkage data from health-related administrative data collections.

Cameron $e t a l^{7} 8$ examined preinjury and postinjury health service use, in terms of admissions and physician claims, from Manitoba for 21000 injured individuals aged 18-64 years with a matched non-injured comparison group while controlling for preinjury comorbid conditions identified from health records. This study found that injured individuals had higher pre-existing comorbid conditions than non-injured individuals ${ }^{7}$ and that after controlling for these comorbidities, those who had been injured experienced higher rates of postinjury health service use than the non-injured, but that only $38.7 \%$ of postinjury hospitalisations in the 10 years following the injury event were attributable to the injury. ${ }^{8}$ Less is known about health service use, the role of comorbidities and attributable injury outcomes for older persons (ie, $\geq 65$ years) and for injured populations in an Australian context. This study aims to quantify 12-month hospitalised morbidity and mortality attributable to traumatic injury, controlling for demographic factors and pre-existing comorbidities, using a $\geq 18$-year population-based matched cohort in Australia.

\section{METHOD}

A population-based matched cohort study of injured and non-injured individuals aged $\geq 18$ years using linked emergency department (ED) presentation, hospitalisation and mortality records from three Australian states from 1 January 2008 to 31 December 2010.

\section{Data sources}

The hospital admission records include information on all inpatient admissions from all public and private hospitals in New South Wales and Queensland and for public hospitals in South Australia. All hospital admission records were included and this contained information on patient demographics, source of referral, diagnoses, external cause(s), hospital separation type (eg, transfer, discharge) and clinical procedures. Diagnoses and external cause codes were classified using the International Classification of Diseases, 10th Revision, Australian Modification (ICD-10-AM).$^{16}$
The ED presentation records contain information collected from public hospital EDs in New South Wales, Queensland and South Australia. Data collected by the EDs included patient demographics, arrival and departure dates, triage category, type of visit and clinical procedures. Mortality data were obtained from the Registry of Births, Deaths and Marriages in the three Australian states. All death information is collected from death certificates (certified by a medical practitioner or pathologist) includes demographic data and date of death.

\section{Injured population}

The injured population was identified if there was a principal diagnosis of injury (ICD-10-AM: S00-T75 or T79) in the hospital admission records in 2009. The first injury-related hospital admission in 2009 was identified as the index injury admission.

\section{Non-injured comparison population}

A population-based, non-injured comparison cohort of individuals aged $\geq 18$ years who were not hospitalised for an injury in 2009 was randomly selected from the electoral rolls in each state. All individuals aged $\geq 18$ years should be registered on the electoral roll as it is compulsory to vote in Australia. Individuals in the non-injured cohort were selected by each state data linkage centre and were matched 1:1 on age, gender, and postcode of residence at the date of the index injury admission of their matched counterpart. Individuals aged $\geq 85$ years were matched using a $\geq 85$ year age group instead of age, if necessary.

All ED, hospital admission and mortality records for the non-injured cohort were identified from 1 January 2008 to 31 December 2010. In New South Wales 182 records and in Queensland 73 records were excluded as no appropriate matched counterpart was able to be identified. Seventeen records were subsequently excluded from Queensland as the gender of the person in the matched comparison population was not the same as their matched injured counterpart.

\section{Data linkage}

Data custodians in each state identified all index injury-related hospitalisations in 2009 in their hospital separation records. Each state-based data linkage centre probabilistically linked all ED, hospitalisation and mortality records of the index injury hospital admissions for individuals with a valid postcode of residence in their state. Each state data linkage centre had access to an extract of the electoral roll and matched counterparts for the index injury cases were randomly identified. All records from New South Wales and South Australia were provided to the Centre for Data Linkage and records for these two states were probabilistically linked to identify any cross-border healthcare use by either injury cases or their matched counterparts. The state linkage centres and the Centre for Data Linkage used identifying information (eg, name, address, date of birth, gender) to 
create a unique identifier for each person identified in the linkage process.

As a result of the cross-border linkage between New South Wales and South Australia, it was identified that 24 individuals from the comparison cohort in South Australia had 59 ED presentations in New South Wales and 9 individuals from the comparison cohort in South Australia were hospitalised 19 times in New South Wales. These ED and hospital admissions in New South Wales were added to the hospital records of the South Australia comparison cohort. There were also 158 records in New South Wales that were removed as these records were identified as members of the comparison cohort in South Australia.

\section{Identification of comorbidities}

The Charlson Comorbidity Index (CCI) was used to identify 17 comorbidities (ie, myocardial infarction, congestive heart failure, peripheral vascular disease, cerebrovascular disease, dementia, chronic pulmonary disease, rheumatological disease, peptic ulcer disease, mild liver disease, diabetes without chronic complications, diabetes with chronic complications, hemiplegia/ paraplegia, renal disease, any malignancy, moderate or severe liver disease, metastatic solid tumour, and AIDS/ HIV) based on diagnosis classifications from the hospitalisation records. ${ }^{17}$ A 12-month look back period from the admission date of the index injury admission was used for the identification of comorbidities for both the injury cases and their matched counterparts. The CCI was treated as a categorical variable and categorised as severe comorbidity $(\mathrm{CCI} \geq 3)$, mild-to-moderate comorbidity (CG1=1 or 2 ) and no reported comorbidity $(\mathrm{CCI}=0){ }^{7}$ In addition, specific health conditions that have been associated with injury risk and poor recovery, ${ }^{18} \quad 19$ including mental health conditions (ICD-10-AM: F20-F50) and alcohol misuse and dependence (ICD-10-AM: F10, Y90, Y91, Z50.2, Z71.4, Z72.1) were also identified using hospitalisation records.

\section{Injury severity}

Injury severity was estimated using the International Classification of Injury Severity Score (ICISS) by applying previously developed survival risk ratios (SRR) to each individual's injury diagnosis classifications (equation 1$).^{20}$ The ICISS is derived for each person by multiplying the probability of survival for each injury diagnosis using SRRs calculated for each injury diagnosis. ${ }^{20}$ Three ICISS levels were used to define minor $(\geq 0.99)$, moderate $(0.941-0.99)$ and serious $(\leq 0.941)$ injury. ${ }^{21}$ For example, a serious injury is equivalent to a survival probability of $94.1 \%$ or a $5.9 \%$ probability of death.

$$
\begin{aligned}
& \mathrm{SRR}_{\mathrm{ICD}_{\mathrm{i}}}= \\
& \frac{\text { Number of individuals with injury } \mathrm{ICD}_{\mathrm{i}} \text { that survived }}{\text { Total number of individuals with injury } \mathrm{ICD}_{\mathrm{i}}}
\end{aligned}
$$

Identification of urban and rural location of residence

The Australian Statistical Geographical Standard Remoteness Area was used to identify rural and urban residents. This classification assigns residents to one of five categories (ie, major cities, inner regional, outer regional, remote and very remote) using defined index scores of distance to service centres of various sizes. ${ }^{22}$ The score is initially calculated on a $1 \mathrm{~km}$ grid, and then the mean value for each Census Collection District is aggregated to form the remoteness areas. The five categories were collapsed into two categories: urban (ie, major cities) and rural (ie, inner regional, outer regional, remote, and very remote).

ED presentations, hospital admissions, hospital length of stay (LOS), and mortality

The number of ED presentations and hospital admissions 12 months preceding and 12 months post the index injury-related admission were identified for both the injured cases and their non-injured counterparts. The calculation of hospital (LOS) 12 months post the index injury admission was cumulative and included transfers between hospitals. Both cumulative hospital LOS and age-adjusted hospital LOS at 12 months post the index injury hospitalisation were truncated to three SDs in order to exclude extreme outliers. ${ }^{23}$ The index injury hospitalisation was not included in the counts of ED presentations, hospital admissions or in the calculation of cumulative hospital LOS. Twelve-month mortality was calculated from the date of admission of the index injury admission.

\section{Data management and analysis}

All analyses were performed using SAS V.9.4 (SAS: statistical software, version 9.4 [program]. Cary, North Carolina, UAS: SAS Institute, 2014). All hospital episodes of care related to the one injury admission (or other hospitalisation for non-injured cohort) were linked to form a period of care (ie, all episodes of care related to the hospitalisation until discharge from the health system). Descriptive statistics were conducted, including $\chi^{2}$ tests of independence and Wilcoxon-Mann-Whitney tests to compare the characteristics of injured individuals admitted to hospital with the comparison cohort, as appropriate. Linear regression was used to age-adjust the hospital LOS post the index injury hospitalisation. Wilcoxon-Mann-Whitney tests were used to compare the injured and non-injured cohorts and unadjusted and age-adjusted hospital LOS. $^{24}$ Mortality counts postinjury excluded records with incongruous dates of hospitalisation and death or the date of death of the non-injured person occurred prior to the date of the index injury hospitalisation resulting in an injury cohort of 166589 individuals with matched controls for the mortality analyses. To calculate the rate per 100000 population of hospital admissions and mortality post the index injury hospitalisation, denominator data for the number of people aged 
$\geq 18$ years residing in New South Wales, Queensland and South Australia were obtained from the Australian Bureau of Statistics population estimates. ${ }^{25}$

Negative binomial regression, adjusted for the number of Charlson comorbidities (ie, 0, 1-2 or 3+), mental health conditions (ie, yes/no) and alcohol misuse and dependence (ie, yes/no) and using the log of survival at 12 months post the index injury as an offset was used to quantify associations between injury and counts of hospital admissions 12-months post the index injury hospitalisation using rate ratios and $95 \%$ CIs. The attributable risk per cent was used to estimate the per cent of hospital admissions where the index injury was a likely contributory factor and was calculated by subtracting 1 from the adjusted rate ratio (ARR), divided by the ARR, multiplied by $100 .^{26}$

\section{RESULTS}

There were 167600 individuals injured in 2009 and admitted to hospital in New South Wales, South Australia or Queensland with a matched non-injured comparison. Men represented $56.9 \%$ of those injured, $29.7 \%$ were aged $18-34$ years, $37.7 \%$ were aged $35-$ 64 years, $32.6 \%$ were aged $\geq 65$ years, and $65.0 \%$ resided in an urban location (table 1). The mean age for the injured and non-injured comparison cohorts was 52.0 years $(\mathrm{SD}=23.3)$. For the injured cohort, the most common injuries were fall related $(38.5 \%)$ and as a result of transport incidents $(12.3 \%)$. The majority of

Table 1 Demographic characteristics of injury-related hospitalisations of individuals aged $18+$ years and matched non-injured comparison cohort in 2009, linked hospitalisation and mortality data in Australia

\begin{tabular}{llc}
\hline & \multicolumn{2}{l}{$\mathbf{n = 1 6 7 6 0 0}$ in each cohort } \\
\cline { 2 - 3 } Characteristic & $\mathbf{n}$ & Per cent \\
\hline Australian state & & \\
$\quad$ New South Wales & 91413 & 54.5 \\
Queensland & 59187 & 35.3 \\
South Australia* & 17000 & 10.1 \\
Gender & & \\
Male & 95333 & 56.9 \\
Female & 72267 & 43.1 \\
Age group & & \\
18-24 & 23350 & 13.9 \\
25-34 & 26399 & 15.8 \\
35-44 & 24175 & 14.4 \\
45-54 & 21465 & 12.8 \\
55-64 & 17672 & 10.5 \\
$65-74$ & 14374 & 8.6 \\
75-84 & 20110 & 12.0 \\
85+ & 20055 & 12.0 \\
Location of residence & & \\
Urban & 109011 & 65.0 \\
Rural & 58589 & 35.0 \\
\hline *Includes people hospitalised in public hospitals in South Australia \\
only.
\end{tabular}

injuries were minor $(43.7 \%)$ or moderate $(37.2 \%)$, with $19.1 \%$ of individuals sustaining serious injuries.

\section{Preinjury hospital service use}

Within the 12 months prior to the index injury hospitalisation of the injured case, $39.8 \%$ of the injured cohort had previously presented to an ED and $30.9 \%$ had been admitted to hospital compared with $15.2 \%$ of the noninjured cohort who had previously presented to an ED and $19.6 \%$ who had previously been admitted to hospital. The majority of injured and non-injured individuals did not have any Charlson comorbidities $(84.4 \%$ and $94.6 \%$, respectively). The injured individuals had a higher proportion of mild-to-moderate (13.1\% vs $5.0 \%)$ and severe $(2.6 \%$ vs $0.3 \%)$ Charlson comorbidities than the non-injured individuals $\left(\chi^{2}=9873.7, \mathrm{df}=2, \mathrm{p}<0.0001\right)$. The proportion of individuals with mental health conditions $(6.2 \%$ vs $0.9 \%)$ and alcohol misuse and dependence $(7.5 \%$ vs $0.4 \%)$ were highest among the injured compared with their non-injured counterparts.

\section{Postinjury hospital service use and mortality}

In the 12 months after the index injury hospitalisation of the injured case, there were significant differences in the number of ED presentations and hospital admissions for the injured and non-injured cohorts, with $36.7 \%$ of the injured cohort presenting to an ED at least once compared with $15.7 \%$ for the non-injured cohort. Six per cent of the injured cohort presented to an ED on four or more occasions in the 12 months following their injury, compared with $1.1 \%$ for the non-injured cohort. Forty-two per cent of the injured cohort had been admitted to hospital in the 12 months following their initial injury compared with $20.0 \%$ of the non-injured cohort, with $4.2 \%$ of individuals admitted on five or more occasions compared with $1.1 \%$ for the non-injured cohort. For those who had been admitted to hospital, there was a significantly longer mean LOS for injured individuals compared with the non-injured cohort, but when age-adjusted the mean LOS was lower for the injured cohort (table 2). The hospitalisation rates of 12 months pre and post the index injury hospitalisation were higher for the injured cohort. The hospitalisation rates were at least twice as high for the injured cohort during the preinjury 12 months and were up to four times as high in the first 2 months following the index injury hospitalisation (figure 1). Mortality in the 12 months post the index injury hospitalisation was higher for the injured compared with the non-injured cohort and sustained across the study period, but was highest in the first 30 days following the index injury hospitalisation (figure 2).

After adjusting for the number of Charlson comorbidities, the injured cohort had twice the rate of hospital admissions post the index injury date compared with the non-injured comparison cohort (ARR 2.34; 95\% CI 2.26 to 2.43). When mental health conditions and alcohol misuse and dependence were also adjusted for, the 
Table 2 Emergency department and hospital admission characteristics and 12-month mortality of injury-related hospitalisations of individuals aged 18+ years and matched non-injured comparison cohort within the 12 months post the index injury hospitalisation, linked hospitalisation and mortality data in Australia

\begin{tabular}{|c|c|c|c|c|c|}
\hline \multirow[b]{2}{*}{ Characteristic } & \multicolumn{2}{|c|}{$\begin{array}{l}\text { Injury cohort } \\
(n=167600)\end{array}$} & \multicolumn{2}{|c|}{$\begin{array}{l}\text { Non-injured } \\
\text { comparison cohort } \\
(n=167600)\end{array}$} & \multirow[b]{2}{*}{$\chi^{2}$ (df) or Z statistic } \\
\hline & $\bar{n}$ & Per cent & $\bar{n}$ & Per cent & \\
\hline \multicolumn{6}{|c|}{ Emergency department presentations in the 12 months post the index injury date } \\
\hline No presentations & 106130 & 63.3 & 141260 & 84.3 & $20935.7(5)^{*}$ \\
\hline 1 presentation & 31813 & 19.0 & 17459 & 10.4 & \\
\hline 2 presentations & 13188 & 7.9 & 5198 & 3.1 & \\
\hline 3 presentations & 6274 & 3.7 & 1895 & 1.1 & \\
\hline 4 presentations & 3492 & 2.1 & 826 & 0.5 & \\
\hline 5 or more presentations & 6703 & 4.0 & 962 & 0.6 & \\
\hline Mean number of presentations (SD) $†$ & 0.9 & $(2.7)$ & 0.3 & $(0.8)$ & $z=-142.9^{*}$ \\
\hline \multicolumn{6}{|c|}{ Hospital admissions in the 12 months post the index injury date } \\
\hline No admissions & 96538 & 57.6 & 134097 & 80.0 & $20639.5(5)^{\star}$ \\
\hline 1 admission & 38046 & 22.7 & 21134 & 12.6 & \\
\hline 2 admissions & 15508 & 9.3 & 6851 & 4.1 & \\
\hline 3 admissions & 7007 & 4.2 & 2648 & 1.6 & \\
\hline 4 admissions & 3477 & 2.1 & 1086 & 0.7 & \\
\hline 5 or more admissions & 7024 & 4.2 & 1784 & 1.1 & \\
\hline Mean number of admissions (SD)† & 1.2 & $(5.3)$ & 0.4 & (3.4) & $z=-143.3^{*}$ \\
\hline \multicolumn{6}{|c|}{ Hospital LOS, cumulative 12 months post the index injury admission (days) } \\
\hline $1-2$ & 29271 & 41.2 & 17723 & 52.9 & $1995.3(5)^{*}$ \\
\hline $3-4$ & 7841 & 11.0 & 3905 & 11.7 & \\
\hline $5-7$ & 6678 & 9.4 & 3232 & 9.7 & \\
\hline $8-14$ & 8310 & 11.7 & 3304 & 9.9 & \\
\hline 15-29 & 8106 & 11.4 & 2724 & 8.1 & \\
\hline $30+$ & 10856 & 15.3 & 2615 & 7.8 & \\
\hline Mean hospital cumulative LOS (SD)† & 16.1 & $(37.1)$ & 9.2 & $(21.6)$ & $z=-44.2^{*}$ \\
\hline Mean age-adjusted hospital cumulative LOS (SD) $†$ & 13.3 & $(5.5)$ & 14.9 & $(5.5)$ & $z=42.5^{\star}$ \\
\hline \multicolumn{6}{|l|}{ 12-month mortality $\ddagger$} \\
\hline Unadjusted & 10638 & 6.4 & 2970 & 1.8 & $4504.8(1)^{*}$ \\
\hline \multicolumn{6}{|c|}{$\begin{array}{l}{ }^{*} p<0.0001 . \\
\text { †Wilcoxon-Mann-Whitney. } \\
\text { †Excludes records with incongruous dates of hospitalisation and death, therefore mortality analyses conducted on an injury cohort of } 166589 \\
\text { individuals with matched controls. } \\
\text { LOS, length of stay. }\end{array}$} \\
\hline
\end{tabular}

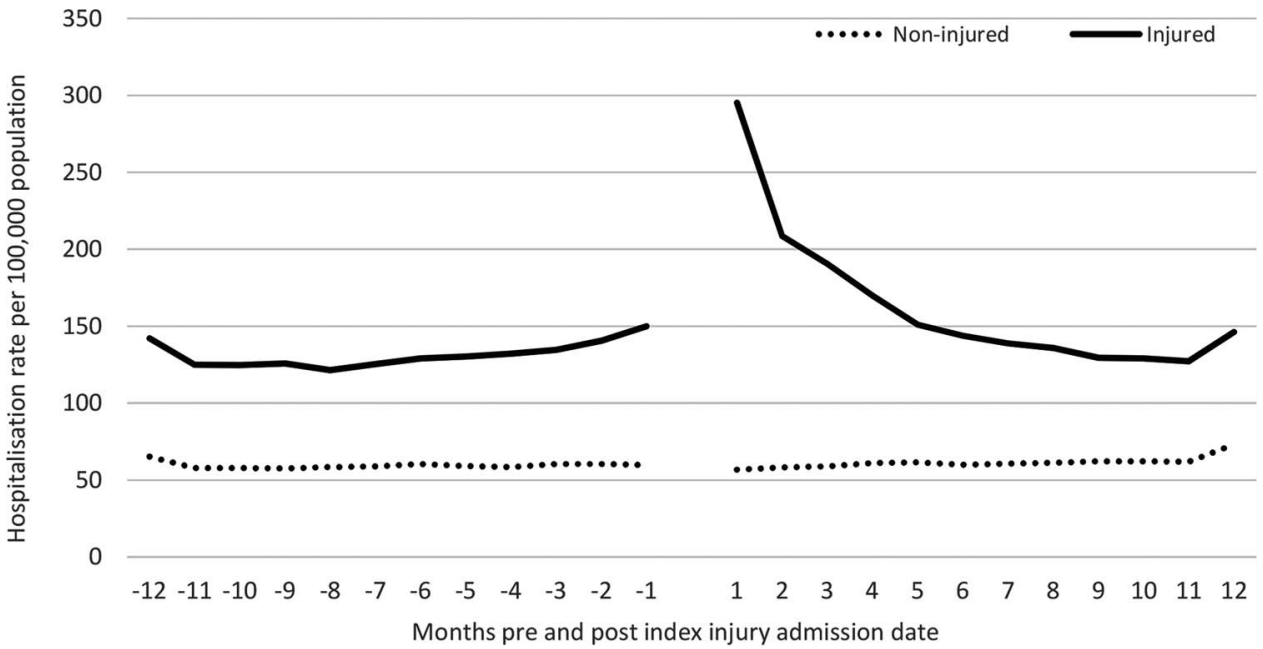

Figure 1 Hospitalisation rates per 100000 population 12 months pre-index and post-index injury hospital admission by month for injured and non-injured comparison group, linked hospitalisation and mortality data in Australia. 


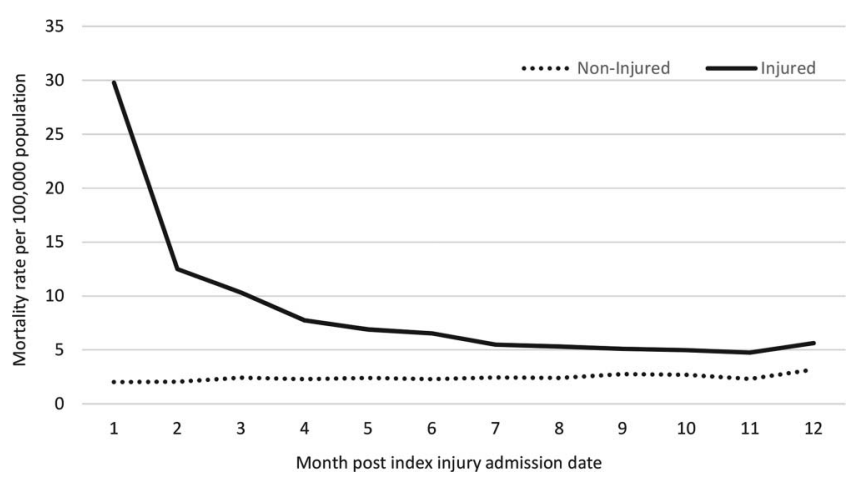

Figure 2 Mortality rates per 100000 population 12 months post-index injury hospital admission by month for injured and non-injured comparison group, linked hospitalisation and mortality data in Australia.

injured cohort had 2.20 (95\% CI 2.12 to 2.28) times higher rate of hospital admissions post the index injury compared with their non-injured counterparts. As age increased, the injured cohort had lower rates compared with their non-injured counterparts. Fifty-five per cent of hospitalisations within 12 months of the index injury were likely to be attributable to the index injury, with attributable risk highest for individuals aged 18-24 years and lowest for those aged $\geq 75$ years (table 3 ).

\section{DISCUSSION}

This study quantified 12-month hospitalised morbidity and mortality attributable to traumatic injury, controlling for demographic factors and pre-existing comorbidities, using a $\geq 18$-year population-based matched cohort in Australia. Injured individuals were found to have higher mortality and more than double the rate of hospital admissions in the 12 months postinjury compared with their non-injured counterparts, after adjusting for the number of Charlson comorbidities, mental health conditions and alcohol misuse and dependence. Over half of all the postinjury hospitalisations were identified as likely to be related to the index injury after taking into account comorbid conditions, with the attributable risk proportion decreasing as age increased. It appears that following the index injury hospitalisation, there is an ongoing burden of care for several months due to injury. The current study findings suggest that Global Burden of Disease and Injury estimates that attribute poor injury outcomes solely to the injury event are potentially overestimating the impact of the injury by not ascertaining the influence of preinjury comorbid conditions, particularly on older age groups.

This study found that the injured cohort had a three times higher proportion of having one or more Charlson comorbidities, a six times higher proportion of mental health conditions and an 18 times higher proportion of alcohol misuse and dependence preinjury compared with the non-injured comparison cohort. Previous research identified that injured individuals experience a five times higher proportion of preinjury comorbidities compared with a non-injured cohort. ${ }^{7}$ However, it is likely that differences in comorbidity ascertainment will account for some of the differences between the studies, with the current study identifying comorbidities from hospitalisation records and the previous study identifying comorbidities using hospital and physician claim records. ${ }^{7}$ Mental health conditions have previously been found to be a common cause of hospitalisation preinjury experienced by an injured compared with non-injured individuals, ${ }^{7}{ }^{27}$ and alcohol use has long been considered to be a risk factor for injury-related hospitalisations. ${ }^{28}$ Interestingly, there was negligible effect of adjusting for mental health conditions and alcohol misuse and dependence for individuals aged $\geq 65$ years, suggesting a likely survival bias, particularly for high-risk alcohol consumers. ${ }^{29}$

This study identified in the 12 months preceding the index injury hospitalisation, the injured cohort had twice the proportion of ED presentations and one and a half times the proportion of hospital admissions compared with their non-injured matched counterparts. Likewise, preinjury hospitalised morbidity was found to be higher in an injured compared with a non-injured comparison group in Manitoba. ${ }^{7}$ In the 12 months after the index injury hospitalisation, the injured cohort had twice the proportion of ED presentations and hospital admissions than their non-injured counterparts. The injured cohort also had three times the proportion of mortality within 12-months of the index injury hospital admission than the non-injured cohort. Similarly, postinjury health service use was found to be higher for the injured compared with non-injured comparison cohorts in Manitoba and in Denmark for each of the 10 years and 9 years, respectively, post the index injury that were examined in each study. ${ }^{810}$ Likewise, a 6-year follow-up of individuals with a spinal cord injury in Alberta during 1992-1994 found that individuals with a spinal cord injury had 2.6 times more re-hospitalisations following their injury compared with a comparison group selected from the general population. ${ }^{9}$ In a study of transport injury in Victoria, Australia from 1995 to 2008, 22.9\% of those who had been injured and hospitalised were found to have reused acute hospital services within the 12 months after their initial hospital discharge. ${ }^{30}$

For the postinjury hospitalisations, there was a significantly longer mean cumulative LOS for injured individuals, but when LOS was age-adjusted, the non-injured cohort had a higher LOS. The difference between the unadjusted and age-adjusted LOS may partly be explained by the non-injured comparison group who were hospitalised having a higher mean age (64.6 years vs 59.0 years) post the injury index hospitalisation compared with the injured cohort. Older individuals, compared with younger, may spend more time in hospital as they are likely to have a higher number of comorbidities, be more physically vulnerable and less likely to physically cope with treatment. ${ }^{31}$ Within the injured cohort, there 


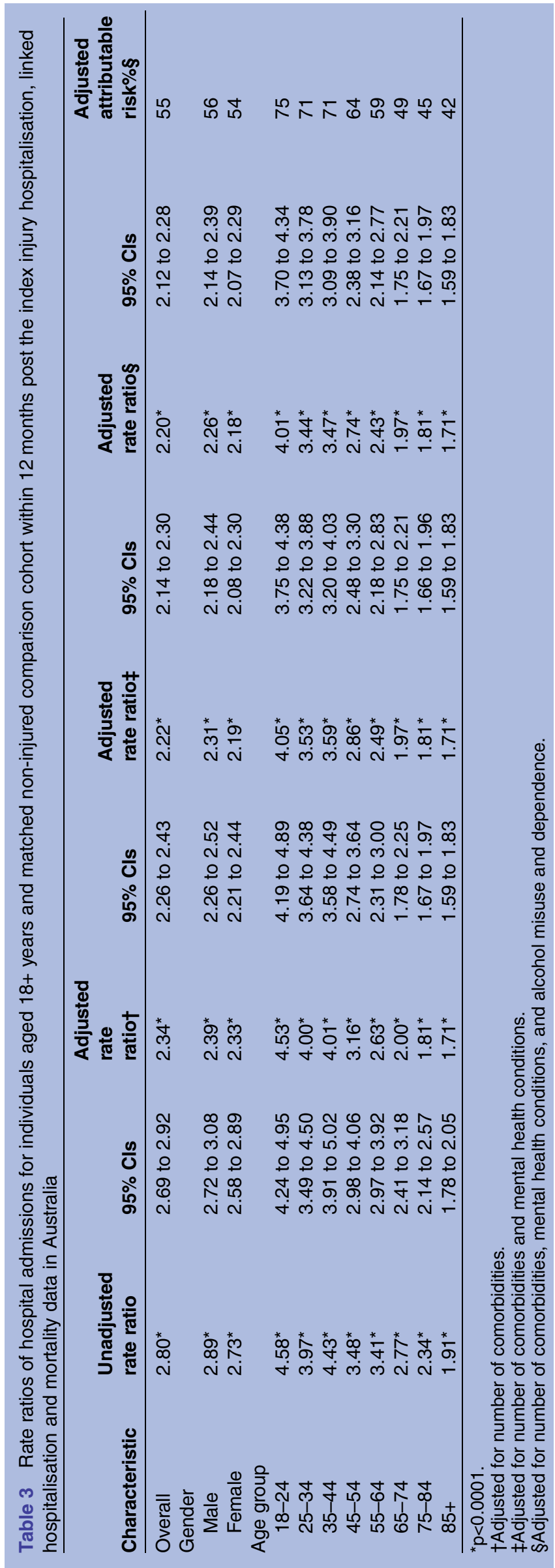

were $48.2 \%$ of women aged $\geq 65$ years compared with $20.7 \%$ of men aged $\geq 65$ years. While men tend to more often be injured in their younger years, ${ }^{32}$ women tend to live longer and are likely to account for the higher proportion of women in this cohort.

There were several limitations associated with the current study. The cross-border record linkage was only able to be conducted in two states, which may have resulted in some individuals residing near the New South Wales-Queensland state border, in particular, using hospital services in either state that may have changed the case-comparison status of an individual or resulted in additional hospital service use being recorded for an individual. No private hospital injury admission data was able to be obtained from South Australia, nor was private hospital ED presentation data able to be obtained. However, $81 \%$ of hospital separations for traumatic injury occur at public hospitals in Australia. ${ }^{33}$ It is possible that equity of access to hospital services and hospital admission policies plaved a role in whether an individual presented and/or was admitted to hospital ${ }^{34}$ and this would have had an impact on injury and comparison cohort selection. This study was a proof of concept that multistate data linkage could be conducted in Australia and when the research began in 2012, the 2010 hospitalisation data was the most recent hospitalisation data available for 12-month follow-up; however, the development of processes, the linkage and provision of correctly linked data extracts to the investigators from all three Australian jurisdictions was only achieved in 2016.

It is compulsory to vote in Australia, but there are some individuals who do not enrol to vote and are missing from the electoral roll, especially young people and also older people who may be incapacitated ${ }^{35}$ and this may have restricted the selection of the comparison cohort. The experience of comorbidities was likely to be under enumerated using hospitalisation data, as only health conditions that were relevant to the current hospital episode of care are reported in each hospitalisation record. However, by using a 1 year look-back period, better estimates of the prevalence of health conditions were able to be obtained. ${ }^{36}$ In addition, there was no information on comorbidities for non-injured individuals who were not hospitalised, resulting in underenumeration of comorbidities likely to be treated by general practitioners or through other health services. The CCI was developed to provide an indicator of survival, rather than of health conditions likely to affect injury-related disability, thus some health conditions, such as mental health conditions or alcohol dependence, likely to affect injury-related disability were not included in the CCI. However, these conditions were adjusted for in this study. The appropriateness of the weightings of the CCI, (especially as a predictor of morbidity rather than mortality) introduces a potential error, but this is likely to be nondifferential in that it affects both samples equally and thus is unlikely to have introduced a bias that threatens the validity of the estimated effects. Data validity was not 
able to be assessed and it is possible there could be some misclassification or inconsistency in classification in records. Finally, when using record linkage, there is likely to be some degree of error in the data linkage process.

\section{CONCLUSION}

The study is one of few that have used a matched cohort design to examine the influence of both comorbidity and injury on postinjury health outcomes. The few relevant studies that have been undertaken suggest that the presence of comorbid conditions can influence recovery and outcomes following an injury and that comorbid conditions are associated with a higher rate of preinjury health service use, particularly as individuals age. The findings of this study provide clear confirmation of previous studies' results. Individuals who had an injuryrelated hospitalisation have higher mortality and are hospitalised at increased rates for at least 4 months postinjury. While comorbid conditions were significant, with higher pre-existing comorbidities and higher preinjury hospital service use, they do not account for the overall group differences. Individuals who are hospitalised for an injury event have significant hospitalised morbidity and increased mortality risk long after discharge from the initial acute care. While the apparent injury-related hospitalisation and mortality is clearly increased for at least 4 months postinjury, morbidity and mortality in the full 12 months after injury is higher in the injured compared with non-injury groups. The study provides a more accurate estimate of a population-based attributable mortality and morbidity following injury thus could contribute to improved quantification of the burden of injury in Australia.

Acknowledgements The authors wish to thank the New South Wales (NSW) Ministry of Health, the Queensland Department of Health and the South Australian Department of Health and Ageing for providing access to their emergency department presentation and admitted patient data collections, the NSW, Queensland and South Australian Registries of Births Deaths and Marriages for providing access to mortality data, and the NSW, Queensland and South Australian electoral commissions for providing access to their electoral rolls. They would also like to thank the Centre for Health Record Linkage (CHeReL), the Statistical Services Branch of the Queensland Department of Health, SA-NT DataLink, and the Centre for Data Linkage for conducting the record linkage. CMC was supported by a Public Health Fellowship (ID 428254) from the National Health and Medical Research Council (NHMRC) Australia.

Contributors RJM, CMC and RM were all involved in study concept and design. RJM acquired the data, conducted the analysis and wrote the first draft of the manuscript. RJM, CMC and RM were all involved in interpretation of data and critical revision of the manuscript.

Funding The research was supported by the Population Health Research Network with funding from the Australian Government National Collaborative Research Infrastructure Strategy and the Western Australian Minister for Science and Innovation.

Competing interests None declared.

Patient consent A waiver of consent was granted by the ethics committees.

Ethics approval Ethical approval was obtained from each Human Research Ethics Committee associated with each State Health Department.
Provenance and peer review Not commissioned; externally peer reviewed.

Data sharing statement No additional data are available.

Open Access This is an Open Access article distributed in accordance with the Creative Commons Attribution Non Commercial (CC BY-NC 4.0) license, which permits others to distribute, remix, adapt, build upon this work noncommercially, and license their derivative works on different terms, provided the original work is properly cited and the use is non-commercial. See: http:// creativecommons.org/licenses/by-nc/4.0/

\section{REFERENCES}

1. Global Burden of Disease Study Collaborators. Global, regional, and national incidence, prevalence, and years lived with disability for 301 acute and chronic diseases and injuries in 188 countries, 1990-2013: a systematic analysis for the Global Burden of Disease Study 2013. Lancet 2015;386:743-800.

2. Lyons RA, Kendrick D, Towner EM, et al. Measuring the population burden of injuries-implications for global and national estimates: a multi-centre prospective UK longitudinal study. PLoS Med 2011;8: e1001140.

3. Australian Institute of Health and Welfare. Australian Burden of Disease Study: impact and causes of illness and death in Australia 2011. Canberra: AlHW, 2016.

4. Derrett S, Langley J, Hokowhitu B, et al. Prospective outcomes of injury study. Inj Prev 2009;15:351.

5. Gabbe BJ, Braff S, Fitzgerald M, et al. RESTORE: REcovery after Serious Trauma-Outcomes, Resource use and patient Experiences study protocol. Inj Prev 2015;21:348-54.

6. Ameratunga S, Norton RN, Whitlock G, et al. The New Zealand blood donors' health study: baseline findings of a large prospective cohort study of injury. Inj Prev 2002;8:66-9.

7. Cameron CM, Purdie DM, Kliewer EV, et al. Differences in prevalence of pre-existing morbidity between injured and non-injured population. Bull World Health Organ 2005;83:345-52.

8. Cameron CM, Purdie DM, Kliewer EV, et al. Ten-year health service use outcomes in a population-based cohort of 21000 injured adults: the Manitoba Injury Outcomes Study. Bull World Health Organ 2006;84:802-10.

9. Dryden DM, Saunders LD, Rowe BH, et al. Utilization of health services following spinal cord injury: a 6 -year follow-up study. Spinal Cord 2004:42:513-25.

10. Laursen B, Helweg-Larsen K. Health service use in adults 20-64 years with traumatic brain injury, spinal cord injury or pelvic fracture. A cohort study with a 9-year follow-up. BMJ Open 2012;2:e001521.

11. Bergeron E, Lavoie A, Moore L, et al. Comorbidity and age are both independent predictors of length of hospitalisation in trauma patients. Can J Surg 2005;48:361-6.

12. Milzman D, Boulanger B, Rodriguez A, et al. Pre-existing disease in trauma patients: a predictor of fate independent of age and injury severity score. J Trauma 1992;30:236-43.

13. Morris JA, McKenzie EJ, Edelstein SL. The effect of pre-existing conditions on mortality in trauma patients. JAMA 1990;263:1942-6.

14. Niven DJ, Kirkpatrick AW, Ball CG, et al. Effect of comorbid illness on long-term outcome of adults suffering major traumatic injury: a population-based cohort study. Am J Surg 2012;204:151-6.

15. Gopinath B, Jagnoor J, Harris IA, et al. Comparison of health outcomes between hospitalised and non-hospitalised persons with minor injuries sustained in a road traffic crash in Australia: a prospective cohort study. BMJ Open 2015;5:e009303.

16. National Centre for Classification in Health. $I C D-10-A M$. 5th edn Sydney: National Centre for Classification in Health, 2006.

17. Quan H, Li B, Couris CM, et al. Updating and validating the Charlson Comorbidity Index and score for risk adjustment in hospital discharge abstracts using data from 6 countries. Am J Epidemiol 2011;173:676-82.

18. Miller TR, Lestina DC, Smith GS. Injury risk among medically identified alcohol and drug abusers. Alcohol Clin Exp Res 2001;25:54-9.

19. Wan J, Morabito D, Khaw L, et al. Mental illness as an independent risk factor for unintentional injury and injury recidivism. J Trauma 2006;61:1299-304.

20. Stephenson S, Henley G, Harrison J, et al. Diagnosis-based Injury Severity Scaling. Adelaide: AIHW, 2003.

21. Dayal S, Wren J, Wright C. Mapping injury severity scores against hospitalisation day stays for injury priority areas (excluding workplace injury). Wellington: Public Health Intelligence, Health and Disability Systems Strategy Directorate, Ministry of Health, 2008. 
22. Australian Bureau of Statistics. 1270.0.55.005-Australian Statistical Geography Standard (ASGS): Volume 5-Remoteness Structure, July 2011. Secondary 1270.0.55.005-Australian Statistical Geography Standard (ASGS): Volume 5-Remoteness Structure, July 201123 July 2014. 2013. http://www.abs.gov.au/AUSSTATS/ abs@.nsf/DetailsPage/1270.0.55.005July\%202011?OpenDocument

23. National Health Performance Authority. Hospital performance: length of stay in public hospitals in 2011-12. Technical supplement. Sydney: National Health Performance Authority, 2013.

24. Armitage P, Berry G, Matthews J. Statistical methods in medical research. 4th edn. Cornwell: Blackwell Science, 2002.

25. Australian Bureau of Statistics. Australian demographic statistics. Cat. no. 3101.0. Canberra: ABS, 2015.

26. Woodward M. Epidemiology: study design and analysis. 2nd edn. Boca Raton: Chapman \& Hall CRC, 2005.

27. Cameron CM, Purdie DM, Kliewer EV, et al. Mental health: a cause or consequence of injury? A population-based matched cohort study. BMC Public Health 2006;6:114.

28. McKenzie K, Harrison JE, McClure RJ. Identification of alcohol involvement in injury-related hospitalisations using routine data compared to medical record review. Aust N Z J Public Health 2010;34:146-52.
29. Tinetti ME, Speechley M, Ginter SF. Risk factors for falls among elderly persons living in the community. N Engl J Med 1988;319:1701-7.

30. Ruseckaite R, Gabbe B, Vogel AP, et al. Health care utilisation following hospitalisation for transport-related injury. Injury 2012;43:1600-5.

31. Wdlinsky FD, Culler SD, Callahan CM, et al. Hospital resource consumption among older adults: a prospective analysis of episodes, length of stay, and charges over a seven-year period. J Gerontol 1994;49:S240-52.

32. Australian Institute of Health and Welfare. Australia's Health 2016. Canberra: AlHW, 2016.

33. Australian Institute of Health and Welfare. Admitted patient care 2013-14. Canberra: Australian Institute of Health and Welfare, 2015.

34. Cryer C, Langley JD. Developing valid indicators of injury incidence for "all injury". Inj Prev 2006;12:202-7.

35. Mitchell R, Cameron C, Bambach M. Data linkage for injury surveillance and research in Australia: perils, pitfalls and potential. Aust N Z J Public Health 2014;38:276-81.

36. Preen DB, Holman CD, Spilsbury K, et al. Length of comorbidity lookback period affected regression model performance of administrative health data. J Clin Epidemiol 2006;59:940-6. 